Universidad de Lima

Facultad de Psicología

Carrera de Psicología

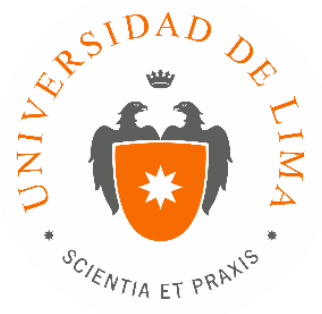

\title{
INTERVENCIÓN DEL DEPARTAMENTO PSICOPEDAGÓGICO DENTRO DE UNA INSTITUCIÓN EDUCATIVA INCLUSIVA PERTENECIENTE AL AREA EDUCATIVA.
}

Trabajo de suficiencia profesional para optar el título profesional de Licenciado en Psicología

Gabriela Milagros Lapeyre Gómez

Código 20052718

Lima-Perú

Febrero 2019 


\section{INTERVENCIÓN DEL DEPARTAMENTO PSICOPEDAGÓGICO DENTRO DE UNA INSTITUCIÓN EDUCATIVA INCLUSIVA PERTENECIENTE AL AREA EDUCATIVA.}




\section{Tabla de contenido}

INTRODUCCIÓN.............................................................................................................. 5

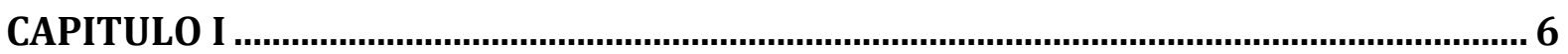

1.1. LA INCLUSIÓN EDUCATIVA DENTRO DEL SISTEMA EDUCATIVO _............................... 6

1.2. AGENTES DENTRO DE UNA INSTITUCIÓN EDUCATIVA.............................................. 7

1.3. DESAFIOS DE LA EDUCACIÓN INCLUSIVA …………................................................ 8

1.4. ESCUELA INCLUSIVA FOMENTANDO EL CAMBIO ……............................................

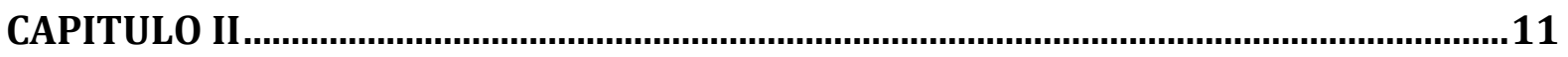

2.1. ACTIVIDADES REALIZADAS HACIA UNA EDUCACIÓN INCLUSIVA .............................11

2.1.1. IDENTIFICACIÓN DE HABILIDADES Y NECESIDADES...................................................... 11

2.1.2. EVALUACIÓN PSICOPEDAGÓGICA ...................................................................................... 12

2.1.3. DETERMINACIÓN DEL PLAN DE ACCIÓN …………………................................................12

2.1.4. REUNIONES PERTINENTES ......................................................................................................

CAPITULO III : RESULTADOS .....................................................................................15

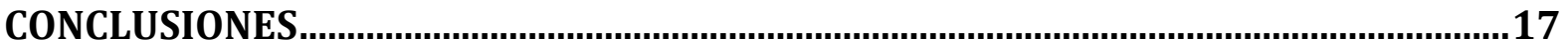

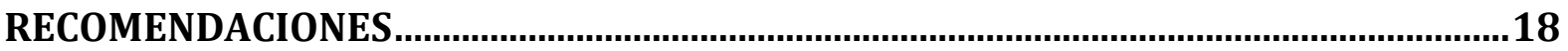

REFERENCIAS......................................................................................................19

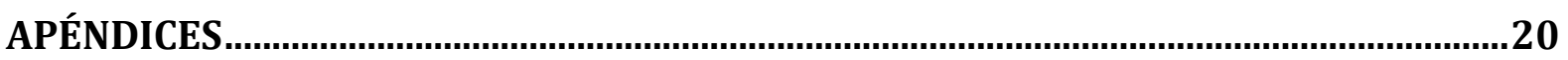

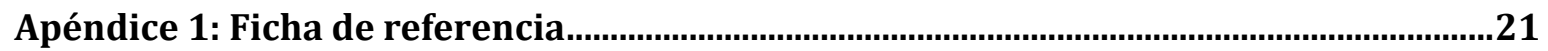

Apéndice 2 : Cuadro de Ajustes Razonables ........................................................................24

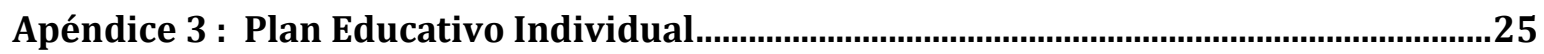

Apéndice 4 : Libreta Diferenciada.........................................................................................27 


\section{INTRODUCCIÓN}

La inclusión ha sido abordada por muchos especialistas dentro del ámbito educativo. Cada día hay mayor disposición y compromiso de los profesionales para realizar nuevas propuestas de trabajo, brindando igualdad de oportunidades a niños con necesidades especiales que desean acceder a una educación regular buscando así eliminar las barreras que dificultan su proceso de aprendizaje.

El punto fundamental para llevar una educación inclusiva adecuada, está en considerar aquellas características particulares que presenta cada niño, enriqueciendo su proceso de enseñanza aprendizaje. Además, es de suma importancia el rol del profesor, quien debe ser una persona preparada para poder brindar espacios que fomenten la autonomía y la reflexión, estimulando y motivando constantemente el logro de los objetivos de cada estudiante.

Por otro lado, podemos visualizar que en los últimos 50 años se han dado cambios que han beneficiado a la inclusión educativa. Por ejemplo, el enfoque tradicional mostraba un estudiante pasivo y sumiso ante el rol del profesor, cambiando está posición a un estudiante respetado por sus aptitudes, brindándole espacios para poder dar lo mejor de sí. También, nos encontramos en un punto donde las escuelas buscan convertirse en una institución transformadora, que intenta adaptarse a estas demandas logrando realizar un trabajo conjunto entre las familias, profesores y especialistas para generar una educación de calidad.

El objetivo de este trabajo de suficiencia profesional es describir las acciones inclusivas realizadas dentro de un centro educativo particular de Lima por parte del departamento psicopedagógico, brindando una propuesta educativa para niños con necesidades especiales buscando participación equitativa e igualdad de oportunidades, basándose en un trabajo multidisciplinar, de acompañamiento y atención a la diversidad dando un giro importante para un educación inclusiva conveniente para el beneficio de los niños. 


\section{CAPITULO I}

\subsection{LA INCLUSIÓN EDUCATIVA DENTRO DEL SISTEMA EDUCATIVO}

El sistema educativo está integrado por diversos factores que pueden ofrecer un ambiente óptimo para cada estudiante. El fin es brindar herramientas a cada uno de ellos para su crecimiento personal y logren así insertarse en la sociedad. La sociedad inclusiva que tanto buscamos nos reta a abrir espacios para liberarnos de las barreras de manera que las personas que tengan dificultades puedan insertarse exitosamente.

En el Perú, de acuerdo a la primera Encuesta Nacional Especializada sobre Discapacidad realizada por el INEI (2014), el 5,2\% de la población total padece de alguna discapacidad, cifra que viene creciendo en el tiempo. De este porcentaje total, el 63,1\% son niños de 6 a 11 años de edad que asisten a un centro educativo y el 50,8\% son estudiantes de 12 a 17 años, todos ellos amparados por la Ley General de la Persona con Discapacidad No 29973 emitida en el año 2012 que tiene como finalidad establecer el marco legal para la promoción, protección y realización, en condiciones de igualdad, de los derechos de la persona con discapacidad, promoviendo su desarrollo e inclusión plena y efectiva en la vida política, económica, social, cultural y tecnológica.

Es por ello, que la educación inclusiva tiende a tomar un rol prioritario buscando favorecer a todos los miembros de la comunidad, implementando metodologías que ayudan a los niños con necesidades especiales (NEE). El Ministerio de Educación (MINEDU, 2012) describe a estos niños como aquellos que presentan dificultades mayores a las del resto de sus compañeros de clase para acceder a los aprendizajes que, de acuerdo al diseño curricular nacional corresponden a su edad, requiriendo adecuaciones en una o varias áreas de su currículo.

El desarrollo de un trabajo inclusivo, requiere considerar la equidad como pieza clave para el éxito de esta tarea, poniendo énfasis en la premisa que "una educación sin equidad, nunca 
será de calidad" (Marchesi y Martín, 2014). Por ello, se hace un replanteamiento de cómo abordar la enseñanza, buscando lograr el nivel máximo de desarrollo de cada alumno dentro de clase, tomando en cuenta sus características personales, sociales y familiares, contrarrestando aquellas necesidades educativas que pueden aparecer de forma permanente o temporal.

Saavedra et al., (2014) define educación inclusiva como "la necesidad de modificar la estructura, el funcionamiento y la propuesta pedagógica para transformar la escuela, con la finalidad de dar respuestas creativas e innovadoras a la diversidad de necesidades que presentan todos y cada uno de los estudiantes". Por ello, para la implementación de una educación inclusiva, es necesario tomar atención a todos los agentes que son participes dentro de esta comunidad, como la plana docente, el departamento psicopedagógico, las familias y las políticas internas de la institución que apoyarán de diversa manera a favorecer el aprendizaje de los niños, fortaleciendo sus cualidades, logrando el desarrollo integral.

\subsection{AGENTES DENTRO DE UNA INSTITUCIÓN EDUCATIVA}

Al constituir un grupo de apoyo para establecer una dinámica activa que cumpla con las condiciones adecuadas para el trabajo inclusivo, es necesario contar con profesionales de calidad que enriquezcan no solo el trabajo académico, sino también fortalezcan aspectos personales y sociales tanto fuera como dentro del aula, con suficiente preparación para reconocer, modificar y ayudar con responsabilidad y empeño.

Calderón (2012) realizó una investigación con el objetivo de identificar los factores que favorecen las relaciones sociales entre niños de aulas inclusivas, encontrando que los maestros cumplen un rol de vital importancia ya que son las personas que tienen el contacto directo conociendo las necesidades particulares, las fortalezas y debilidades logrando un mejor manejo. De esto podemos concluir que los maestros son las personas encargadas de guiar y plantear nuevas estrategias para brindar un ambiente adecuado que fomente la 
diversidad, generando un clima emocional apropiado, así como de elaborar material oportuno que sea de apoyo para los estudiantes regulares como aquellos niños con necesidades especiales.

Otro agente dentro de la escuela son los psicólogos, quienes ayudan a realizar la evaluación, diagnóstico y acompañamiento para las dificultades de aprendizaje y emocionales. Son las personas que brindan asesoramiento tanto a profesores como familias, colaborando de manera apropiada por el bienestar de los niños. Los psicólogos prestan atención a las demandas solicitadas, recogiendo, analizando e interpretando los resultados de las evaluaciones para poder realizar la toma de decisiones y la elaboración de programas especializados que se realizará en el año escolar. Además, proporciona un apoyo consistente ante cualquier incidente y mantiene el trabajo conjunto entre los agentes para llevar acabo las acciones pertinentes para el proceso de enseñanza aprendizaje.

Por último, las familias brindan esencial apoyo, su participación e involucramiento en el aprendizaje de los niños es básico para el fortalecimiento y desarrollo de nuevos desafíos. Existen estudios como el de Tellado y Sava (2010) que demuestran que la participación de los padres en la escuela genera actitudes positivas y desarrollan habilidades que progresan en el tiempo. El involucrar a miembros de la familia hace que los niños tengan mayor motivación hacia el objetivo progresando eficientemente en el tiempo.

\subsection{DESAFIOS DE LA EDUCACIÓN INCLUSIVA}

En el transcurso de la implementación de una educación inclusiva, las escuelas tienden a transformar la cultura, políticas y prácticas que se realizan. Asimismo, tienen la misión de sensibilizar a todos los involucrados que están dentro de este proceso desarrollando una tarea compleja que puede traer consigo retos que pueden intervenir de manera negativa si no se realiza un buen control. 
Uno de los principales desafíos que presenta la educación inclusiva es la resistencia y la falta de capacitación por parte de los maestros. Ellos deben comprender la condición que presentan sus alumnos y brindar estrategias que fomenten oportunidades. Sin embargo, la falta de información, compromiso y poca minuciosidad en los detalles puede interrumpir el desempeño de los niños NEE generando realidades poco favorable para el desarrollo de sus habilidades.

El poco involucramiento de las familias, también genera un retroceso en el aprendizaje. Los niños que no reciben apoyo constante y consistente tienden a no alcanzar los objetivos planteados, siendo perjudicial para su crecimiento. Por ello, es importante ser constantes, brindando el apoyo y condiciones adecuadas para su desarrollo.

Por otro lado, actualmente la sociedad está iniciando un cambio, ya que busca romper estereotipos, pero aún hay una falta de educación orientada hacia la sensibilidad y empatía. El eliminar estas barreras implica un cambio de mentalidad, que genere menor resistencia al cambio, esto requiere un trabajo exhaustivo para buscar la manera de educar a la sociedad, produciendo una modificación en el estilo de vida para que todos los niños tengan acceso a una educación apropiada con igualdad de oportunidades basada en sus propias habilidades.

\subsection{ESCUELA INCLUSIVA FOMENTANDO EL CAMBIO}

El presente trabajo de sustentación profesional está basado en acciones realizadas dentro del colegio particular Newton College, que tiene como misión ser reconocido a nivel mundial por su excelencia académica y estilo educativo, teniendo como visión desarrollar al máximo el potencial de sus alumnos formando ciudadanos íntegros y exitosos. De este modo, toma en cuenta las necesidades educativas individuales, valorando la diversidad y aplicando la diferenciación en las clases de acuerdo al perfil del alumno.

El colegio cuenta con un departamento psicopedagógico que se encuentra comprometido con brindar asistencia a todos los niños que requieran apoyo, considerando que todos los 
estudiantes de una u otra forma requieren de atención. El equipo psicopedagógico posee un manual de políticas de necesidades educativas especiales para niños con discapacidad y dificultades de aprendizaje, haciendo adaptaciones curriculares y acomodaciones en el trabajo que se realiza en el día a día. Además, realiza un acompañamiento de la mano con especialistas externos y familias buscando el bienestar integro de sus estudiantes. 


\section{CAPITULO II}

\subsection{ACTIVIDADES REALIZADAS HACIA UNA EDUCACIÓN INCLUSIVA}

Una psicóloga del colegio Newton College, tiene como principal objetivo conocer con minuciosidad las habilidades y áreas de mejora de cada uno de los niños NEE, con el fin de poder darle la mayor cantidad de recursos que faciliten el logro de aquellas metas planteadas en un plan individual que favorezca el desarrollo de sus capacidades. En este camino, encontramos un grupo, cada vez mayor, con necesidades educativas especiales, el cual es atendido siguiendo un protocolo establecido.

Las actividades realizadas en la intervención especializada dentro del colegio son:

1.-) Identificación de Habilidades y necesidades.

2.-) Evaluación Psicopedagógica.

3.-) Determinación del plan de acción.

4.-) Reuniones pertinentes.

\subsubsection{IDENTIFICACIÓN DE HABILIDADES Y NECESIDADES}

El colegio posee tres niveles de educación, inicial, primaria y secundaria. Es una población heterogénea, integrada por estudiantes de 2 a 18 años, muchos de ellos con necesidades temporales y otros permanentes (Ministerio de Educación, 2013). Algunos de los casos con NEE temporales dentro del colegio son: niños desmotivados, niños en proceso de duelo, falta de seguimiento de instrucciones, regulación de emociones, niños extranjeros, etc. Algunos de los casos con NEE permanentes son: niños con Déficit de Atención e Hiperactividad, niños con Coeficiente Intelectual Limítrofe, niños con Trastorno del Espectro Autista, niños con Trastorno Específico de Lenguaje, etc.

La principal función del psicólogo es conocer a los alumnos, identificando sus fortalezas y debilidades, estilos de aprendizaje, dificultades familiares, etc. Esta información es 
compartida con tutores y profesores que tienen contacto con los estudiantes, reservando datos propios de la confidencialidad.

Una vez iniciado el proceso escolar y luego de profundas observaciones, evaluaciones, etc. los profesores refieren a niños que poseen alguna necesidad dentro o fuera de clase ya sea académica, social o emocional, para ello llenan una ficha de referencia (Apéndice 1) que es enviada vía web al departamento Psicopedagógico. El objetivo de conocer las habilidades y necesidades de los alumnos es poder realizar un trabajo de más específico, adecuado a las características de cada niño, brindándole un ambiente apropiado para la convivencia y el aprendizaje.

\subsubsection{EVALUACIÓN PSICOPEDAGÓGICA}

El objetivo de realizar una evaluación psicopedagógica es poder describir, identificar y explicar el perfil del niño, analizando posibles causas de su comportamiento. El rol de la psicóloga no sólo será la aplicación de las pruebas, sino también consistirá en poder brindar con una base sólida y científica, posibles planes de acción y herramientas que serán de utilidad para el trabajo diario con el alumno de necesidades educativas especiales.

Estas evaluaciones nos ayudan a medir variables como la atención, personalidad, inteligencia, memoria, madurez visomotora, nivel académico, etc.

Los test empleados en este proceso dentro del colegio son: el Test de la Figura Humana, Dibujo de la Familia, Dibujo Libre, Caras, Cumanes, Bender, Wisc IV, Frases Incompletas, entre otros.

La psicóloga de grado, toma la decisión de solicitar evaluaciones externas para contrastar y confirmar la presunción diagnóstica para el inicio y ejecución de un plan de acción.

\subsubsection{DETERMINACIÓN DEL PLAN DE ACCIÓN}

La determinación de un plan de acción, se aplica de acuerdo a los niveles de concreción curricular (Ministerio de Educación, 2013), el colegio cuenta con tres niveles de atención. En 
el primer nivel, es el tutor quien atiende las necesidades de los estudiantes siguiendo el Currículo Nacional otorgado por el Ministerio de Educación.

En el segundo nivel de atención, se realizan acomodaciones y adaptaciones según las necesidades del alumno. Estos apoyos se plasman en un cuadro de ajustes razonables (Apéndice 2) que permiten tener una visión clara de hacia donde se desea llevar al niño.

En el tercer nivel existe una programación que atiende a la diversidad, ajustando las actividades de acuerdo a las características del niño y donde existe un plan individualizado de atención. Este Plan Educativo Individual (PEI) (Apéndice 3) incluye las fortalezas y debilidades del niño, objetivos personales, medidas de organización, metas esperadas y otras consideraciones que ayudan a realizar un seguimiento en el tiempo. El desarrollo de este PEI se realiza en compañía de la especialista de aprendizaje que es parte del equipo psicopedagógico.

En el caso de los padres con niños NEE, al finalizar el trimestre, reciben una libreta diferenciada (Apéndice 4) que se elabora con el tutor de clase, la especialista de aprendizaje y la psicóloga del grado, mostrando cualitativamente los avances, cambios y futuros logros que el niño debe obtener.

El psicólogo tiene la responsabilidad de tomar las decisiones para la atención que se requiera apoyando a los niños en las dificultades que presentan, por ejemplo, pautas y estrategias a los profesores sobre el trabajo de clase, recomendaciones para los padres de familia de crianza o autonomía, sugerencias para modificación de conducta, fortalecimiento de vínculo, consejos sobre relaciones interpersonales, etc.

\subsubsection{REUNIONES PERTINENTES}

En el desarrollo de todas estas acciones, para buscar la inclusión de niños con necesidades especiales, el papel del psicólogo es mantener constante comunicación con todas las personas que impactan en el desarrollo académico, emocional, familiar y social del niño, con ello se 
busca dar una respuesta a los apoyos brindados, facilitando la intervención logrando una educación integrada y consistente para cada alumno.

Según lo publicado por Ascue (2014) en la revista .edu, un contexto educativo y familiar comprensivo y adecuado pueden hacer diferencia en los logros del niño, desarrollando todas sus potencialidades y habilidades. Es por ello, que dentro de la institución, la psicóloga tiene como función programar, coordinar y asistir a reuniones periódicas con la familia pudiendo calmar aquellas preocupaciones que puedan atravesar durante el año escolar, además de informar posibles cambios en los planes de acción y los logros obtenidos por trimestres.

El acompañante terapéutico es un especialista formado en manejo conductual que pueda hacer de puente para ayudar a coordinar el trabajo de la profesora, la psicóloga, los especialistas y los padres, logrando la inserción del niño con necesidades especiales al ámbito escolar con seguridad y confianza. Con esta persona se tienen reuniones semanales para obtener y compartir información sobre el desenvolvimiento del niño con NEE, se ven los avances, se proponen cambios de estrategias, de ser necesario, así como posibles intervenciones. También, se analizan los efectos sobre el cambio de medicación, entre otros puntos, con el fin de generar el sentido de pertenencia dentro de su ámbito educativo. 


\section{CAPITULO III : RESULTADOS}

Es conveniente resaltar la importancia de poder brindar un ambiente acogedor con oportunidades para que los niños con NEE logren objetivos deseados.

De acuerdo a la identificación de habilidades, el 100\% de los niños con NEE referidos han sido atendidos por la psicóloga, conociendo a profundidad la realidad en donde crecen, siendo una táctica valiosa ya que de ese total, el $88,89 \%$ de ellos han logrado integrarse adecuadamente a su rutina diaria en el colegio, asegurando que el vínculo es necesario para el cambio.

La población seleccionada está constituida por 109 alumnos de segundo de primaria que de acuerdo a los niveles de atención está conformado por, 66,97\% niños en nivel 1, el 27,52\% de alumnos están ubicados en el nivel 2 y 5,5\% son niños con necesidades especiales, nivel 3. Considerando que el nivel 2 y 3 son atendidos por el departamento psicopedagógico, podemos concluir que el 94,5\% de este grupo de alumnos han realizado una evaluación psicopedagógica completa.

Asimismo, los planes de acción y el desarrollo del mismo (reuniones, seguimiento y supervisión) han sido productivos para el aumento de sus capacidades. La psicóloga ha realizado un $78 \%$ de consejería con familias, $36,1 \%$ de apoyo con especialistas y el 33,33\% con acompañantes terapéuticos, observando mejoras académicas visualizadas en su desempeño, emocionales con mayor seguridad e independencia e interpersonales con una mejor adaptación (dependiendo del caso) de un 77,78\% en todos los niños de nivel 2 y 3 . Este logro se evidencia también por el reconocimiento de las familias quienes se encuentran agradecidas con el apoyo brindado por parte del colegio, ya que observan a sus hijos más independientes, reflexivos y con capacidad para resolver problemas y tareas que van acorde con sus necesidades. 
Por otro lado, corroborando lo que menciona Ascue (2014) sobre el apoyo de las familias y especialistas, cuando se realiza un trabajo constante y orientado hacia los objetivos se construye hacia un propósito, evidenciando esto con el paso de una niña de nivel 3 a nivel 2, demostrando que estimulando las áreas necesarias se puede lograr metas esperadas.

Además, sobre los apoyos entregados a las profesoras, se ha observado un incremento del uso de las estrategias propuestas por la psicóloga, siendo esto reflejado por la disminución de las llamadas de emergencia que las profesoras realizan en el día a día.

Si bien, es el inicio de un proceso de cambio en la educación hacia una escuela inclusiva, es importante continuar con este proyecto ya que es de gran ayuda y soporte para aquellas personas que tienen la misma necesidad de pertenecer a una sociedad que aún le cuesta aceptar el desarrollo neurotípico y atípico de los chicos.

Este trabajo sirve como aporte para imitar aquellos comportamientos que son propicios para una educación inclusiva, en escuelas que inician un cambio pensado en la sensibilización y igualdad de oportunidades. 


\section{CONCLUSIONES}

Luego del trabajo realizado, podemos brindar conclusiones que ayudan a mejorar la inclusión de niños con necesidades educativas especiales dentro del entorno educativo.

- Las características particulares de cada niño son el eje principal de un trabajo inclusivo dentro del centro educativo.

- Es indispensable el involucramiento de la familia en el proceso de enseñanza aprendizaje de un niño NEE. Mientras más una familia este involucrada con el desarrollo de su hijo, se observan mejoras y logros en el tiempo.

- El maestro cumple un rol primordial para el logro de los objetivos planteados para un niño NEE, debe mostrarse abierto a los cambios, tener mucha disposición y comprometerse con cada acción que realizará. De él depende una buena adaptación dentro del entorno escolar.

- Es necesario estar vigilante del desempeño y comportamiento del niño NEE, existen factores externos al educativo que pueden influir en su modo de actuar dentro del colegio.

- El vínculo es indispensable para un mejor trabajo con niños NEE.

- Los alumnos que pertenecen al colegio Newton College tienen la oportunidad de contar con los recursos económicos para tener una atención de calidad y multidisciplinar, que muchos niños en la misma condición (nivel 2 y 3 ) en el Perú no la tienen.

- Los alumnos son afortunados de pertenecer a una institución, como el Newton College, que tiene el interés de poder brindar material de calidad, atención con profesionales calificados y mucha entrega por dar lo mejor de sí por el bienestar del niño. 


\section{RECOMENDACIONES}

- Propiciar una mayor cantidad de capacitaciones para los profesores que tienen contacto directo con los niños NEE. Algunos de ellos aún muestran poca flexibilidad realizando una educación tradicional que beneficia poco a niños que necesitan una atención diferenciada.

- El colegio podría promover grupos de apoyo para padres con hijos NEE, con el fin de que esta familias puedan aliviar sus preocupaciones y sentimientos pudiendo expresar sus emociones y compartir sus experiencias, sintiéndose acompañados en todo momento.

- Crear un Foro o blog para la comunidad educativa, en donde cada semana profesores, psicólogos y directivos, puedan publicar experiencias personales con estrategias útiles para tener un banco de recursos que se pueden usar para el manejo de los niños NEE.

- Impulsar talleres para colegios que estén iniciando un proceso de inclusión educativa, en donde el departamento de psicopedagogía del colegio Newton College pueda enseñar su metodología de trabajo, compartir su manual de políticas y brindar ejemplos de los logros obtenidos en estos años que se ha venido trabajando con niños NEE. Con ello se busca expandir los conocimientos y contar con mayor cantidad de especialistas en trabajo inclusivo. 


\section{REFERENCIAS}

Ascue, S. (1 de Abril de 2014). El Autismo y la inclusión en la educación [Mensaje de un blog] Recuperado de https://puntoedu.pucp.edu.pe/opinion/el-autismo-y-la-inclusionen-la-educacion/

Calderón, M. (2012). La Educación inclusiva es nuestra tarea. Educación, 11(40), 43-58.

Congreso de la República (13, diciembre 2012). Ley General de la Persona con Discapacidad.[ Ley 29973 ]. Recuperado de https://www.mimp.gob.pe/webs/mimp/herramientas-recursos-violencia/contenedordgcvg-recursos/contenidos/Legislacion/Ley-general-de-la-Persona-conDiscapacidad-29973.pdf

Instituto Nacional de Estadistica e Informatica (2014). Primera Encuesta Nacional Especializada sobre Discapacidad 2012. Recuperado de https://www.inei.gob.pe/media/MenuRecursivo/publicaciones_digitales/Est/Lib1171/ ENEDIS\%202012\%20-\%20COMPLETO.pdf

Marchesi, A . y Martín, E . (2014) . Calidad de la enseñanza en tiempos de crisis. Madrid: Alianza editorial .

Ministerio de Educación del Perú [MINEDU]. (2012). Educación básica especial y educación inclusiva. Balance y perspectiva [Versión PDF]. Recuperado de http://www.minedu.gob.pe/minedu/archivos/a/002/05-bibliografia-para-ebe/9educacion-basica-especial-y-educacion-inclusiva-balance-y-perspectivas.pdf

Ministerio de Educación del Perú [MINEDU]. (2013). Guía para orientar la intervención de los servicios de apoyo y asesoramiento para la atención de las necesidades educativas especiales SAANEE [Versión PDF]. Recuperado de http://www.minedu.gob.pe/minedu/archivos/a/002/05-bibliografia-para-ebe/7-guiapara-orientar-la-intervencion-de-los-saanee.pdf

Saavedra, M ${ }^{\circ}$.E., Hernández, A., y Ortega, L. D. (2014). Estudio de caso de dos experiencias ganadoras del III Concurso Nacional Experiencias Exitosas en Educación Inclusiva - 2010 (Tesis de Maestria). Pontificia Universidad Católica del Perú, Lima, Perú. Recuperado de http://tesis.pucp.edu.pe/repositorio/bitstream/handle/123456789/5524/SAAVEDRA_ HERNANDEZ_ORIEGA_ESTUDIO_INCLUSIVA.pdf?sequence=1\&isAllowed=y

Tellado, I. Y Sava, S. (2010). The role of non-expert adult guidance in the dialogic construction of knowledge. Revista e Psicodidáctica, 15(2), 163-176. 


\section{APÉNDICES}




\section{Apéndice 1: Ficha de referencia}

\section{HOJA DE REFERENCIA}

Alumno(a):

Edad:

Grado:

Tutor:

Para poder brindar el apoyo adecuado a los alumnos, por favor completa la información. Por favor, llena OBLIGATORIAMENTE cada sección con todas tus observaciones(Marcar al menos una opción)

MOTIVO POR EL CUAL SE REFIERE AL ALUMNO

\section{Comportamiento}

( ) Se para constantemente

Comentarios :

( ) No obedece

( ) No cumple con las tareas asignadas

( ) Es desafiante

( ) Pelea con los amigos

( ) No participa

( ) Sale del salón

Habilidades motoras gruesas - fina

( ) Mala postura corporal

Comentarios :

( ) Movimientos descoordinados

( ) Tonicidad muscular débil o fuerte.

( ) Tiene letra ilegible

( ) Agarra el lápiz inadecuadamente

( ) Trazos débiles

( ) No se observa ninguna dificultad 


\section{Aprendizaje}

( ) No cumple con sus tareas

Comentarios :

( ) No comprende las indicaciones orales y escritas

( ) Ritmo lento de trabajo

( ) Se distrae fácilmente

( ) Tiene dificultades en aprendizajes nuevos

( ) Muestra desorganización

( ) Dificultades en la escritura

\section{Lenguaje}

\begin{tabular}{|l|l}
\hline ( ) No articula las palabras correctamente & Comentarios:
\end{tabular}

( ) Muestra dificultades al estructurar sus ideas

( ) Interpreta las situaciones literalmente

( ) Ecolalias

( ) Tartamudez

( ) No hace inferencias, comparaciones, análisis y síntesis

\section{Aspecto social y emocional}

( ) Poco asertivo

( ) Poco empático

( ) Tiene dificultades para resolver conflictos

( ) Baja tolerancia a la frustración

( ) Ansiedad

( ) No se integra

( ) Es inseguro
Comentarios:

\section{Situación familiar}

( ) Padres separados/divorciados

Comentarios :

( ) Vive con abuelos 
( ) Huérfanos

( ) Familiares enfermos

\section{MEDIDAS TOMADAS PARA BRINDAR APOYO DENTRO DEL SALÓN}

( ) Reducir nivel de lenguaje o lectura

Comentarios :

( ) Usar textos alternativos

( ) Utilizar manipulativos, visuales y colores

( ) Usar organizadores gráficos

( ) Verificar el entendimiento del estudiante

( ) Amigo cooperativo

( ) Tiempo extendido

( ) Instrucciones en distintos pasos pequeños

( ) Dividir texto en secciones

( ) Uso de calculadora

( ) Uso de computadora

\section{Condición Médica especial}

\begin{tabular}{|l|l|}
\hline ( ) Epilepsia & Comentarios: \\
( ) Diabetes & \\
( ) Enfermedad Coronaria & \\
( ) Aspoglicemia & \\
\hline
\end{tabular}

ATENCIÓN O APOYO EXTERNO

Nombre del especialista:

Teléfono y correo:

Medicación (Frecuencia):

Especificar Terapia:

Clases Particulares: 
Apéndice 2 : Cuadro de Ajustes Razonables

\section{AJUSTES RAZONABLES}

\begin{tabular}{|l|l|l|}
\hline Nombre: & Clase: & Fecha de inicio: \\
\hline Grado: & Staff involucrado: & Fecha de cierre: \\
\hline \multicolumn{2}{|l}{} \\
\hline
\end{tabular}

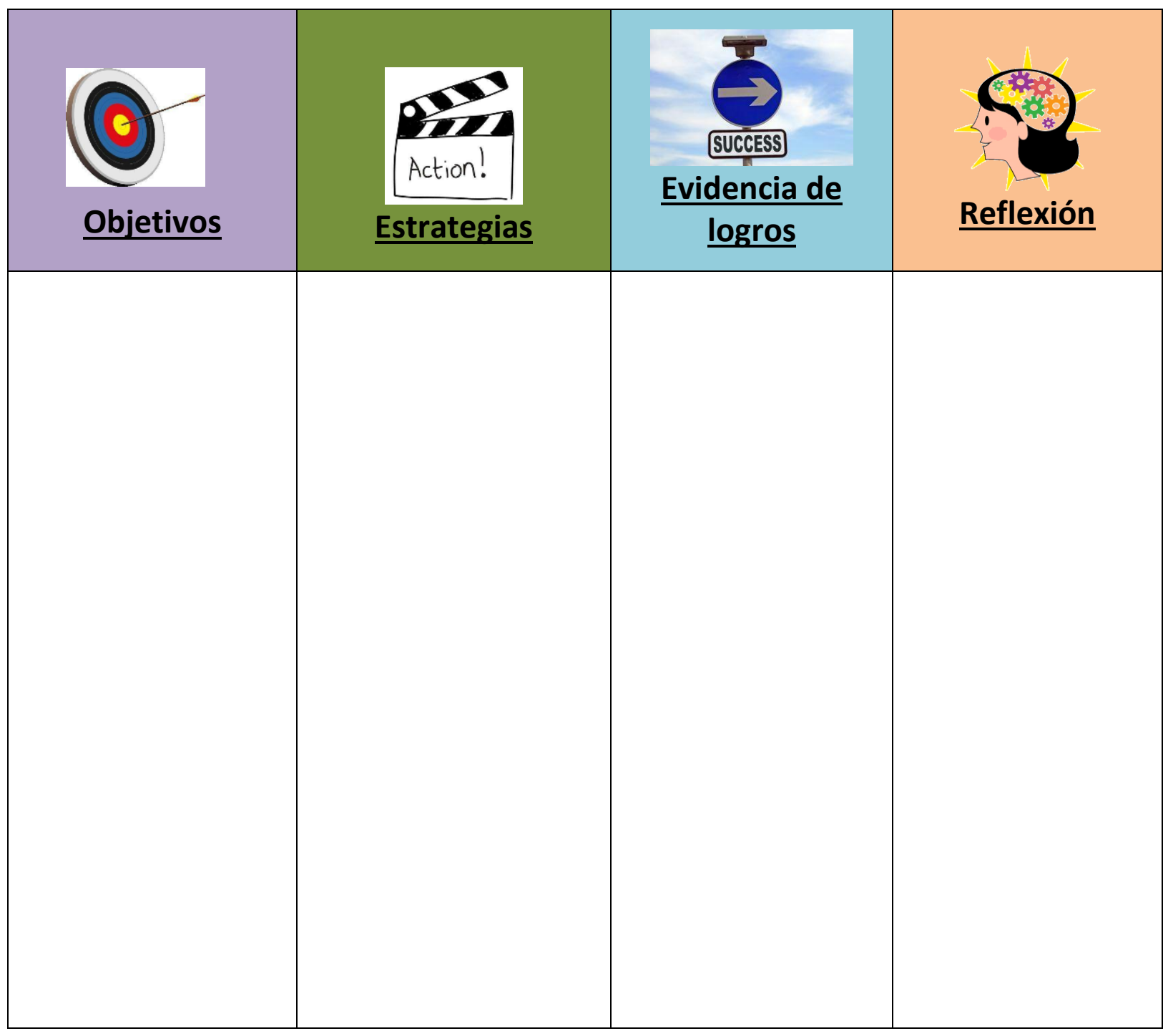




\section{Apéndice 3 : Plan Educativo Individual}

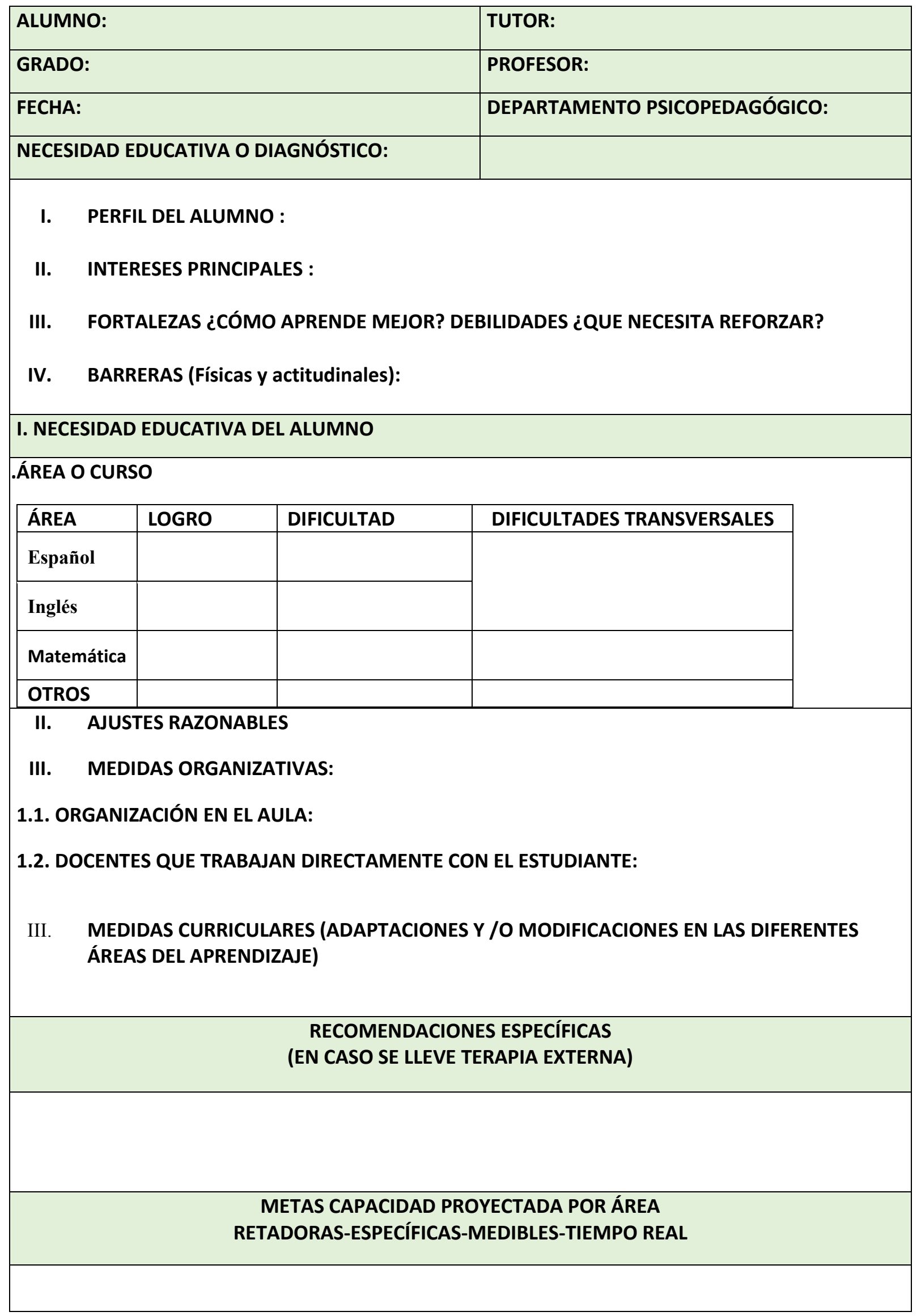




\begin{tabular}{|c|}
\hline EVIDENCIA EN EVALUACIONES \\
\hline CÓMO SE MEDIRÁN SUS PROGRESOS HACIA LOS OBJETIVOS \\
\hline \\
\hline SEGUIMIENTO \\
\hline \\
\hline
\end{tabular}

Firma de los padres

Firma de Dpto Psicoped.

Fecha: 


\section{Apéndice 4 : Libreta Diferenciada}

\section{NEWTON COLLEGE-LOWER SCHOOL DIFFERENTIATED REPORT-Marking Period}

Name:

Class:

In certain courses, some students are not assessed according to the criteria used for the Grade Level. Alternative criteria are applied, taking into consideration the student's individual abilities and needs. The alternative assessment criteria for your son /daughter are listed below.

En determinados cursos, algunos estudiantes no son evaluados de acuerdo a los criterios usados para el grado. Tomando en consideración las habilidades y necesidades de estos estudiantes, hemos utilizado criterios de evaluación alternativos. El criterio de evaluación alternativo de su hijo/hija está explicado abajo.

\section{Subject}

\begin{tabular}{|c|c|c|c|}
\hline \multicolumn{2}{|c|}{ A-Di } & B-Di & C-Di \\
\hline $\begin{array}{c}\text { Developing } \\
\text { Area }\end{array}$ & Oral and visual \\
communication & & & \\
\hline Reading & & & \\
\hline Lriting & & & \\
\hline & & & \\
\hline
\end{tabular}




\begin{tabular}{|l|l|l|l|}
\hline $\begin{array}{l}\text { Approaches to } \\
\text { Learning }\end{array}$ & A-Di & B-Di & C-Di \\
\hline Creative & & & \\
\hline Collaborative & & & \\
\hline Reflective & & & \\
\hline Independent & & & \\
& & & \\
\hline
\end{tabular}
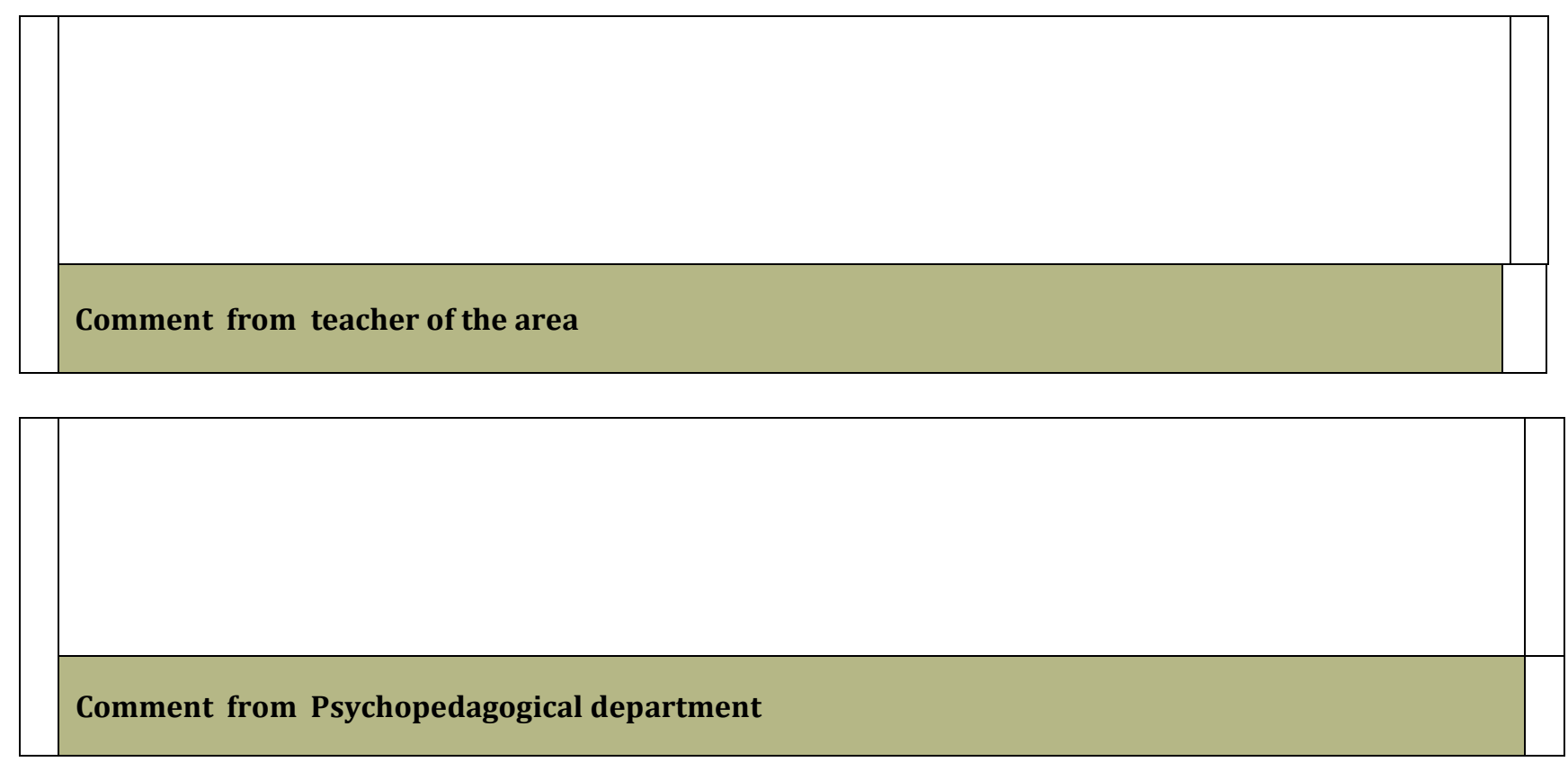